\title{
Identification of polyketide synthase gene clusters in a phage P1- derived artificial chromosome library of a Philippine strain of Streptomyces sp. PCS3-D2
}

\author{
Aileen Bayot Custodio ${ }^{a}$ and Edwin Plata Alcantara ${ }^{a^{*}}$ \\ ${ }^{a}$ National Institute of Molecular Biology and Biotechnology, University of the Philippines Los Baños, Laguna, Philippines 4031
}

Received 5th January 2019 / Accepted 11th March 2019

\begin{abstract}
A phage P1-derived artificial chromosome (PAC) library was constructed from genomic DNA of Streptomyces sp. PCS3-D2. Polymerase chain reaction (PCR) screening of the PAC library revealed two clones, PAC16D and P222O, which were positively identified to harbor polyketide synthase (PKS) Type I and PKS Type III gene clusters, respectively. Restriction enzyme digestion showed that PAC16D and PAC222O contained a $130 \mathrm{~kb}$ and a $140 \mathrm{~kb}$ insert, respectively. Results of sequencing and bioinformatics analyses revealed that PAC16D comprised of a full-length PKS type I bafilomycin gene cluster while PAC222O harbored truncated siderophore and putative gene clusters as well as a complete PKS III biosynthetic gene cluster. The PKS III gene cluster had three genes similar to alkyl resorcinol biosynthetic genes, however majority of the novel gene cluster had little similarity to known PKS Type III gene clusters. The successful cloning and identification of these gene clusters from Streptomyces sp. PCS3-D2 serve as the jump off point to further genetic manipulation in order to produce the insecticidal natural product in a heterologous host.
\end{abstract}

Keywords: polyketide synthase gene clusters, Streptomyces sp. PCS3-D2, phage P1-derived artificial chromosome (PAC) library, natural products

\section{INTRODUCTION}

Actinobacteria are ubiquitous Gram-positive, filamentous bacteria that could be easily isolated from various soil and marine environments. They are well known prolific producers of bioactive compounds including natural insecticides (Kirst, 2010). The use of natural products as insecticides for crop protection is encouraging. Recent reports indicate that the use of natural products and natural product-derived insecticides continue to increase (Dayan et al., 2009). In 2012, natural products and natural product-inspired products comprised approximately $30 \%$ of the global agrochemical sales, while an additional $22 \%$ of synthetic agrochemical compounds could have been inspired by natural products under different circumstances (Gerwick and Sparks, 2014).

The strain Streptomyces sp. PCS3-D2 produces insecticidal compounds active against important agronomic insect pests (Bayot-Custodio et al., 2014). The development of potentially beneficial natural products from this strain is hindered by the lack of proper genetic characterization which when ignored could lead to large missed opportunity for developing effective solutions for mitigating problems in insect resistance to insecticides. Recent statistics showed that at least 30 cases of insecticide resistance have been reported for the Philippines (www.pesticide

* Author for correspondence: Edwin Plata Alcantara, National Institute of Molecular Biology and Biotechnology, University of the Philippines Los Baños, Laguna, Philippines 4031. Email - epalcantara@up.edu.ph 
resistance.org).

The bottom-up approach for natural product discovery utilizes genome sequencing and bioinformatics to identify gene clusters predicted to synthesize important bioactive compounds. Upon identifying a target gene cluster, genetic manipulation techniques are employed to activate the gene clusters either in native or heterologous hosts for the production of the corresponding natural product (Luo et al., 2014). Given that the size of some gene clusters can exceed $100 \mathrm{~kb}$, artificial chromosome-based vectors have been constructed for the maintenance and genetic manipulation of natural producers in Streptomyces (Alduina and Gallo, 2012).

Phage P1-derived artificial chromosomes (PACs) can carry large 100-300 kb DNA fragments in E. coli cells (Shizuya et al., 1992). However, because E. coli is not suitable to heterologously express high $\mathrm{GC} \%$ actinomycete genes, shuttle vectors have been designed to allow library construction and manipulation in E. coli, while the expression of actinomycete genes are done in Streptomyces host strains. An example of this is the pESAC13 vector, which contains the phage P1 origin of replication, phiC31 integrase gene and a phiC31 attP site, an oriT site that allows transfer into Streptomyces by conjugation (Sosio et al., 2000) and a BamHI cloning site for the insertion of large DNA fragments (Jones et al., 2013).

In this paper, we first report the construction of a phage P1-derived artificial chromosome library and the in silico characterization of two cloned polyketide gene clusters from a Philippine strain of Streptomyces sp. PCS3-D2 using a bioinformatics approach.

\section{MATERIALS AND METHODS}

\section{Genome mining of Streptomyces sp. PCS3-} D2 for biosynthetic gene clusters. The previously annotated genome sequence of Streptomyces sp. PCS3-D2 (Bayot-Custodio et al., 2014) was re- analyzed using the antiSMASH 4.0 software (Blin et al., 2017) to determine both the presence and location of biosynthetic gene clusters in the JDUZ01 genome sequence.
Cell culture for construction of phage P1 artificial chromosome (PAC) library. For preparing mycelia for PAC library construction, Streptomyces sp. PCS3-D2 was grown in YMB (100 $\mathrm{mL}$ ) that was inoculated with mycelia from a 10 $\mathrm{mL}$ TSB starter culture. The mycelia were grown for 72 hours with shaking at ambient temperature and centrifuged $\left(4^{\circ} \mathrm{C}, 12000 \mathrm{rpm}, 15 \mathrm{~min}\right)$ to remove the supernatant. The mycelial pellets (approximately $7 \mathrm{~mL}$ of concentrated mycelia) were flash frozen in liquid nitrogen and shipped in dry ice to BioS\&T (Montreal, Canada) for custom construction of a phage P1 artificial chromosome (PAC) library using a modified E. coli-Streptomyces Artificial Chromosome (ESAC) vector (Fig. 1). The constructed Streptomyces sp. PCS3-D2 PAC library generated a total of 2,688 clones with an average insert size of $125 \mathrm{~kb}$.

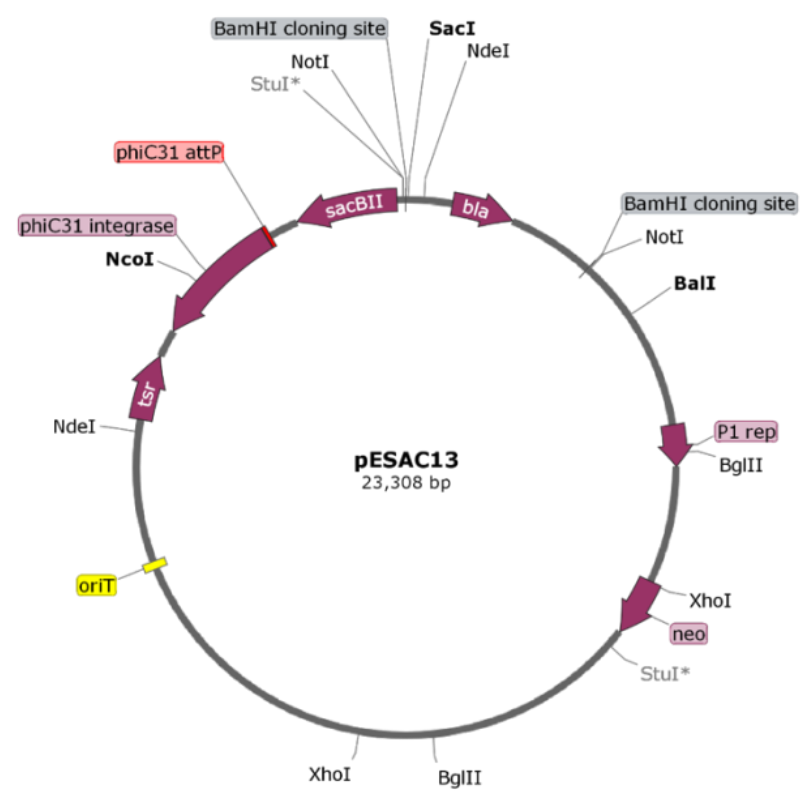

Figure 1. Vector map of PAC pESAC13.

Screening of PAC Library for PKS I and PKS III gene clusters. PCR primers (Table 1) were designed to screen the Streptomyces sp. PCS3-D2 PAC library for clones containing PKS I and PKS III gene clusters. These PCR primers were initially tested using Streptomyces sp. PCS3-D2 genomic DNA as template for PCR. PKS I-specific primers were expected to amplify a $700 \mathrm{bp}$ amplicon corresponding to the 5-aminolevulinate synthase gene of the bafilomycin gene cluster. On the other hand, a 450 bp PCR amplicon was expected to be amplified using PKS III-specific primers corresponding to the stilbene synthase 
gene. Each PCR amplicon was later sequenced to verify that the desired region of the gene clusters was amplified.

The Streptomyces sp. PCS3-D2 PAC library was PCR screened by BioS\&T (Montreal, Canada) using the designed PKS I and PKS III-specific primers. The positively identified clones were subjected to restriction enzyme digestion using NotI to estimate the size of the cloned inserts. Positively identified clones were subjected to next generation sequencing to determine if they contained the full-length PKS I and PKS III gene cluster.

Table 1. Bacterial strains, vectors and PCR primers used in the study.

\begin{tabular}{|c|c|c|}
\hline Items & Description & Reference \\
\hline Streptomyces sp. PCS3-D2 & Wildtype strain producing insectidal compounds & This work \\
\hline Escherichia coli DH10B & $\begin{array}{c}\text { Cloning strain; F- mcrA } \Delta \text { (mrr-hsdRMS-mcrBC) } \\
\varphi 80 \text { lacZ } \Delta \text { M15 } \Delta \text { lacX74 recA1 endA1 araD139 } \Delta \text { (ara, } \\
\text { leu) } 7697 \text { galU galK } \lambda \text { - rpsL nupG }\end{array}$ & Invitrogen \\
\hline pESAC13 Vector & $\begin{array}{l}\text { PAC vector (P1-phage replicon) for genomic library } \\
\text { construction; RP conjugative, } \Phi C 31 \text { integrative tsr, neo, } \\
\text { oriT, attP and int from } \Phi C 31, \mathrm{P} 1 \text { rep, sacB }\end{array}$ & $\begin{array}{l}\text { Sosio et al., } 2000 \\
\text { Genbank No. } \\
\text { LM999999.1 }\end{array}$ \\
\hline BafBIII-F & TCCGAGATCAGCGTGTGGTGC & This work \\
\hline BafBIII -R & GCSGTGGTGAAGATGAAGGRGC & This work \\
\hline STS-F & TTCCCGCCCCACCGCCATTCCCAG & This work \\
\hline STS-R & ACACRGCTCGGTGGACAGCAGCAG & This work \\
\hline
\end{tabular}

\section{Sequencing, assembly, annotation and} analysis of PAC clones. To ensure that the positive PAC clones contained the entire PKS I and PKS III gene clusters, they were subjected to Ion Torrent single ended sequencing with $>200$ coverage depth using the Ion PGM ${ }^{\mathrm{TM}}$ System by BioS\&T (Montreal, Canada). The resulting raw sequence reads were quality checked using FastQC (Andrews, 2018). The sequences were processed by trimming low quality ends using the Trim Ends algorithm of the Geneious R11 software (Kearse et al., 2012). The sequence reads were also screened against the UniVec database to trim off contaminating vector and adapter sequences at the 5' and 3' ends. The filtered and trimmed sequences were subjected to reference guided mapping and assembly using the Geneious R11 software. The Bowtie2 version 2.3.0. plugin (Langmead and Salzberg, 2012) was used in reference guided assembly to align the sequencing reads to the reference sequence. The reference sequence used was scaffold 1 (scf1) of Streptomyces sp. PCS3-D2 JDUZ01 genome sequence. The consensus sequence from reference guided mapping was annotated using the Prokka Galaxy Tool Version: 1.12.0 (Seemann, 2014). The annotated gene clusters were then analyzed using antiSMASH 4.0 for the identification, annotation and analysis of secondary metabolite biosynthesis gene clusters (BGC).

\section{RESULTS}

Genome mining of Streptomyces sp. PCS3D2 for biosynthetic gene clusters. The antiSMASH 4.0 software enables bacterial genome mining for biosynthetic gene clusters that code for chemical classes of secondary metabolites (Blin et al., 2017). This is done by comparing each gene product in the uploaded DNA sequence against a manually curated collection of profile hidden Markov models (pHMMs) which describe key biosynthetic enzymes of the secondary metabolite classes. The key enzymes encoded in each gene cluster are assigned to secondary metabolite-specific clusters of orthologous groups (smCOGs). Depending on the class of secondary metabolite gene cluster detected (i.e. multimodular polyketide synthases (PKSs), non-ribosomal peptide synthetases (NRPSs), etc.), further detailed analyses are performed (Blin et al., 2013). KnownClusterBlast is also integrated in antiSMASH which allows the identification of gene clusters against a repository of known and experimentally characterized secondary metabolite biosynthesis gene clusters called MIBiG (Minimum Information on Biosynthetic Gene Cluster) for comparative gene cluster analysis (Blin et al., 2017). 
The antiSMASH 4.0 analysis revealed that JDUZ01 genome sequence has a total of 26 BGCs which includes polyketide synthases (PKS), nonribosomal peptide synthetases (NRPS), NRPS hybrids, terpenes, siderophores, bacteriocin, butyrolactone, lantipeptides, ectoine, nucleoside and other BCGs as summarized in Table 2. The $\%$ similarity from antiSMASH results was based on how similar the query sequences were with known and characterized gene clusters in the
$\mathrm{MIBiG}$ repository. Among the PKS gene clusters, two were identified as type I PKS: cluster 14 which has high similarity $(100 \%)$ to the bafilomycin gene cluster and cluster 3 that has low homology $(11 \%)$ to PKS-Cf saccharide herboxidiene BGC. Cluster 19, which is probably involved in the synthesis of spore pigments, was classified as a type II PKS, while cluster 13 has strong similarity $(100 \%)$ to type III PKS alkyl resorcinol gene cluster.

Table 2. List of predicted secondary metabolite biosynthetic gene clusters in Streptomyces sp. PCS3-D2 based on antiSMASH 4.0 analysis.

\begin{tabular}{cccc}
\hline Cluster & Type & Predicted Products & \% Similarity \\
\hline 1 & Bacteriocin & Unknown & - \\
2 & Terpene & Unknown & - \\
3 & Type I PKS-Cf saccharide & Herboxidiene & $11 \%$ \\
4 & Terpene-NRPS & Hopene & $61 \%$ \\
5 & NRPS & Streptolydigin & $10 \%$ \\
6 & NRPS & Bleomycin & $6 \%$ \\
7 & NRPS & Streptothricin & $87 \%$ \\
8 & Ectoine & Ectoine & $100 \%$ \\
9 & Thiopeptide-Terpene-NRPS & Coelichelin & $100 \%$ \\
10 & Terpene & 2-methylisoborneol & $100 \%$ \\
11 & Melanin-Terpene & Istamycin & $4 \%$ \\
12 & Siderophore & Kedarcidin & $1 \%$ \\
13 & Type III PKS & Alkylresorcinol & $100 \%$ \\
14 & Type I PKS & Bafilomycin & $100 \%$ \\
15 & Butyrolactone & Neocarzinostatin & $6 \%$ \\
16 & Lantipeptide & Galbonolides & $6 \%$ \\
17 & Lantipeptide & Venezuelin & $100 \%$ \\
18 & NRPS & Tambromycin & $100 \%$ \\
19 & Type II PKS & Spore pigment & $66 \%$ \\
20 & NRPS & Unknown & - \\
21 & Terpene & Carotenoid & $63 \%$ \\
22 & Siderophore & Desferrioxamine B & $100 \%$ \\
23 & Nucleoside & Unknown & - \\
24 & Siderophore & Unknown & - \\
25 & NRPS & Unknown & - \\
26 & NRPS & Enduracidin & $6 \%$ \\
\hline & & &
\end{tabular}

PCR screening of Streptomyces sp. PCS3-D2

$\boldsymbol{P A C}$ library. The PKS I primers amplified a 700 bp fragment of the 5-aminolevulinate synthase gene of the bafilomycin gene cluster (BafBIII) while PKS III primers amplified a 450 bp portion of the stilbene synthase gene (STS) which represents PKS III gene clusters. PCR-based screening of the PAC library identified a clone in plate 1-6D (designated as PAC16D) to harbor the
PKS I gene cluster and another in plate 2-22O (designated as PAC222O) to contain the PKS III gene cluster. Figure $2 \mathrm{a}$ illustrates the PCR screening of PAC16D amplifying a 700 bp PKS I gene product while PAC222O produced the expected $450 \mathrm{bp}$ PKS III band. A $130 \mathrm{~kb}$ and 140 $\mathrm{kb}$ digest were observed from the NotI-treated PAC16D and PAC222O, respectively (Figure 2b). 


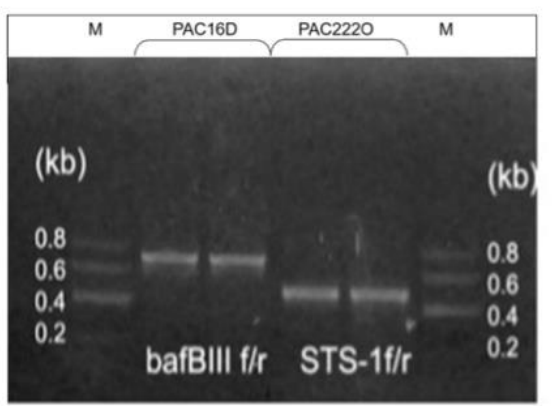

(A)

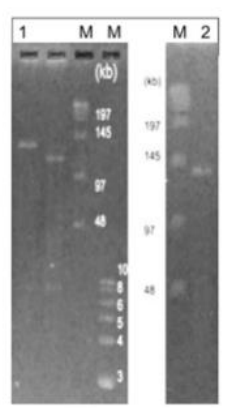

(B)
Figure 2. PCR screening of the Streptomyces sp. PCS3-D2 PAC library for (A) PKS I (PAC16D) and PKS III (PAC222O) gene clusters and (B) PAC clone insert verification by NotI restriction enzyme digestion. Lane 1: $130 \mathrm{~kb}$ PAC16D insert; Lane 2: $140 \mathrm{~kb}$ PAC222O insert; M: molecular weight markers.

\section{$P A C$ clone sequencing and bioinformatics} analysis. Genome sequence analysis of JDUZ01 scf1 showed that it contained 14 gene clusters as illustrated in Figure 3. PKS III and PKS I BGCs were determined to be located near the 3' end of JDUZ01 scf 1 . The PKS III gene cluster was estimated to be located at $1,457,140$ to $1,492,845$ $\mathrm{bp}$, having a total size of approximately $35.7 \mathrm{~kb}$. On the other hand, the PKS I gene cluster was located at 1,621,560 bp to $1,713,412$ bp with a total length of about $91.8 \mathrm{~kb}$.

Next Generation Sequencing generated 246,357 and 181,775 raw read sequences for PAC16D and PAC222O, respectively. Based on the assembly results, PAC222O had an insert length of about $132 \mathrm{~kb}$ which spanned a portion of the neighboring siderophore gene cluster, full length PKS III gene cluster and a putative gene cluster downstream of PKS III (Figure 3, green line). This shows that PAC222O contained the full length PKS III gene cluster.

On the other hand, PAC16D had a $130 \mathrm{~kb}$ insert which included a portion of a putative gene cluster upstream of the PKS I gene cluster as well as the full length PKS I bafilomycin gene cluster (Figure 3, red line). This proves that PAC16D also contains the entire PKS I gene cluster.
Bioinformatics analysis using the antiSMASH 4.0 software revealed that PAC16D had high similarity with the PKS I bafilomycin gene cluster. PAC16D contained the modular polyketide synthases which were responsible for the synthesis of bafilomycin's polyketide backbone, bafilomycin regulatory genes, and other genes responsible for the production and conversion of bafilomycin derivatives (Figure 4a). PAC16D was also compared with known bafilomycin gene clusters from Streptomyces griseus DSM 2608, S. lobii JCM 14114 and Kitasatospora setae KM-6054. Pairwise alignment revealed that PAC16D had $83.9 \%$ similarity with $S$. lobii JCM 14114, 81.6\% with $S$. griseus DSM 2608 and $77.2 \%$ with $K$ setae KM-6054 bafilomycin gene clusters. This proves that PAC16D contains the full length PKS I bafilomycin gene cluster. The best way to confirm that PAC16D contains a bafilomycin gene cluster that is capable of producing bafilomycin is to transform the PAC16D clone into a Streptomyces heterologous host and check for the production of bafilomycin by HPLC or LC-MS.

$\mathrm{PAC} 222 \mathrm{O}$, on the other hand contained three gene clusters: a truncated siderophore gene cluster (red box), the PKS III gene cluster (tan box) and a putative gene cluster (navy blue box) (Figure 4b). The truncated siderophore gene cluster was about $12 \mathrm{~kb}$ in length and included nine genes (Figure 4c). Downstream from the siderophore gene cluster was the full-length PKS III gene cluster (Figure 4d). A neighboring putative gene cluster that overlapped with the 3' end of the PKS III gene cluster was also observed in PAC222O. The PKS III gene cluster has three genes that has high similarity $(100 \%)$ with $S$. griseus subsp. griseus NBRC 13350 alkyl resorcinol genes (monooxygenase, methyltransferase and stilbene synthase genes) (red box). This proves that PAC222O contains a PKS III alkyl resorcinol gene cluster. However, the genes surrounding the PAC222O PKS III gene cluster itself were not similar with other known alkyl resorcinol gene clusters and could therefore be a novel producer of resorcinol derivatives. 


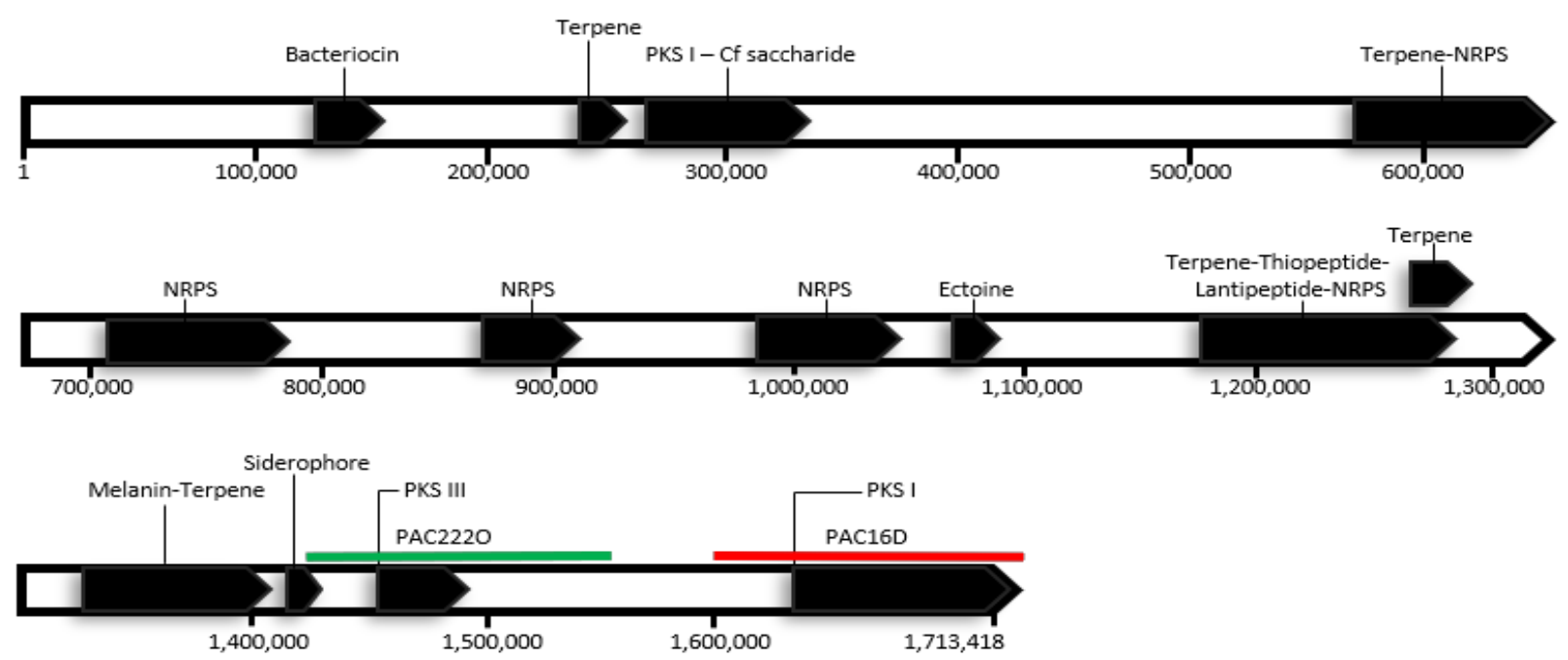

Figure 3. Mapping of biosynthetic gene clusters in JDUZ01 scaffold 1 of Streptomyces sp. PCS3- D2. The green line represents the location of the PAC222O insert while the red line represents the insert of PAC16D. (Cf-saccharide: Putative saccharide cluster; NRPS: Nonribosomal peptide synthetase; PKS I: Polyketide synthase Type I; PKS III: Polyketide synthase Type III).

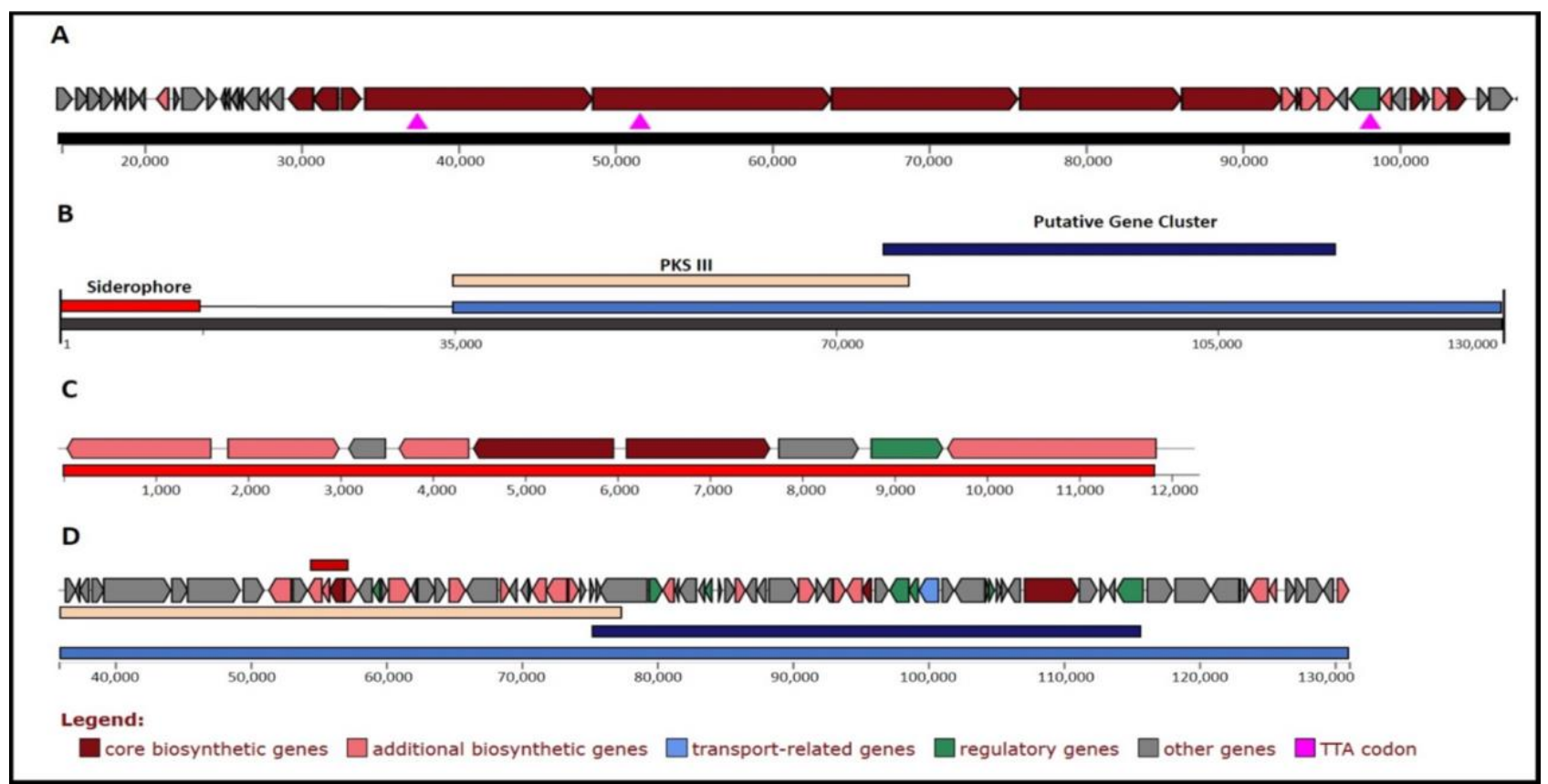

Figure 4. antiSMASH prediction of biosynthetic gene clusters of Streptomyces sp. PCS3-D2 PAC clones (A) PAC16D and (B) PAC222O; (C) truncated siderophore gene cluster in PAC222O; and (D) segment of PAC222O showing the PKS III gene cluster and an overlapping putative gene cluster. The red box represents genes with high similarity to alkyl resorcinol biosynthetic genes.

\section{DISCUSSION}

In this study, genome mining and construction of a PAC library was carried out in order to gain insight into possible ways of exploiting the diversity of BGCs present in Streptomyces sp. PCS3D2. The whole genome sequence of Streptomyces sp. PCS3-D2 served as a blueprint to identify which gene clusters should be prioritized for screening in the PAC library. Indeed, more than twenty-five BGCs were discovered some of which might be responsible for production of novel insecticidal secondary metabolites in Streptomyces sp. PCS3-D2. The discrepancy in the present number of predicted BGCs as compared to our 
previous results (Bayot-Custodio et al., 2014) might be due to modifications made in the antiSMASH 4.0 software (Blin et al., 2017) used in this study.

The PKS I and PKS III BGCs were screened out from the PAC library because from the list of predicted BGCs (Table 2), we infer that there was a high possibility that these two types of PKS might be responsible for production of insecticidal compound in Streptomyces sp. PCS3D2. Several variants of bafilomycin have been reported to have insecticidal activity (Kretschmer et al., 1985). To the best of our knowledge, insecticidal alkyl resorcinol has not been reported from any Streptomyces strains although insecticidal counterpart had been reported from plant origin (Kwon et al., 1996). Other previously discovered insecticides from Actinomycetes are also produced by PKS gene clusters (Waldron et al., 2000; Ikeda et al., 1999; Sun et al., 2003). The PKS type II gene cluster in Streptomyces sp. PCS3-D2 was identified to be involved in spore pigment synthesis and was therefore not included in the PCR screening.

Closer analysis of the PKS III BGC showed that only three of the PKS III genes (monooxygenase, methyltransferase and stilbene synthase) had high similarity to the alkyl resorcinol biosynthesis genes. The rest of the PKS III gene cluster did not show similarity to any known PKS Type III gene cluster, making it a potentially novel PKS Type III gene cluster.

Screening of the Streptomyces sp. PCS3-D2 PAC library resulted to the identification of desired clones with insert size up to $140 \mathrm{~kb}$. Cloning of large DNA fragments of Actinomycetes BGCs with sizes up to $150 \mathrm{~kb}$ has been reported previously (Alduina et al., 2005; Dai et al., 2011). Because the complete PKS I and PKS III gene clusters were present in PAC16D and PAC222O, they are good candidates for heterologous expression to increase yield of insecticidal compound.

\section{CONCLUSION}

Genome mining facilitated the identification of 26 biosynthetic gene clusters including type I and type III polyketide synthases (PKSs) which corresponds to a bafilomycin and alkyl resorcinol gene cluster, respectively. These two PKS gene clusters were successfully screened out from a constructed PAC library of Streptomyces sp. PCS3D2. Heterologous expression will allow confirmation of insecticidal activity being conferred to Streptomyces sp. PCS3-D2 by either or both these two PKS gene clusters.

\section{ACKNOWLEDGEMENT}

The authors thank the Department of Agriculture-Philippine Agriculture and Fisheries Biotechnology Program (DA-BIOTECH) for funding this research. We also thank Bea Crisostomo, Felaine Anne Sumang and Rachelle Leah Melendrez for their assistance with this project. The Streptomyces sp. PCS3-D2 isolate was kindly provided by Teofila Zulaybar (Antibiotics Laboratory, BIOTECH, University of the Philippines Los Baños).

\section{REFERENCES}

Alduina R., Giardina A., Gallo G., Renzone G., Ferraro C., Contino A., Scaloni A., Donadio S., \& Puglia A. 2005. Expression in Streptomyces lividans of Nonomuraea genes cloned in an artificial chromosome. Applied Microbiology and Biotechnology 68 (5): 656-662.

Alduina R. \& Gallo G. 2012. Artificial chromosomes to explore and exploit biosynthetic capabilities of Actinomycetes. Journal of Biomedicine and Biotechnology.

Andrews, S. 2018. FastQC: A quality control tool for high throughput sequence data http://www.bioinformatics. babraham.ac.uk/projects/fastqc/. Accessed February 10 2018.

Bayot-Custodio A. N., Alcantara E. P., \& Zulaybar T. O. 2014. Draft genome sequence of insecticidal Streptomyces sp. strain PCS3-D2, isolated from mangrove soil in the Philippines. Genome Announcement 2(3): e00448-14 .

Blin, K., Medema, M. H., Kazempour D., Fischbach, M. A., Breitling, R., Takano E., \& Weber T. 2013. antiSMASH 2.0 - a versatile platform for genome mining of secondary metabolite producers. Nucleic Acids Research 41 (W1): W204W212.

Blin, K., Wolf, T., Chevrette, M. G., Lu, X., Schwalen, C. J., Kautsar, S. A., Suarez Duran, H. G., De Los Santos, E. L. C., Kim, H. U., Nave, M., Dickschat, J. S., Mitchell, D. A., Shelest, E., Breitling, R., Takano, E., Lee, S. Y., Weber, T., \& Medema, M. H. 2017. AntiSMASH 4.0- improvements in chemistry prediction and gene cluster boundary identification. Nucleic Acids Research 45(W1): W36-W41. 
Dai S., Ouyang Y., Wang G., \& Li X. 2011. Streptomyces autolyticus JX-47 Large-insert bacterial artificial chromosome library construction and identification of clones covering geldanamycin biosynthesis gene cluster. Current Microbiology 63(1): 68-74.

Dayan F., Cantrell C., \& Duke S. 2009. Natural products in crop protection. Bioorganic \& Medicinal Chemistry 17(12): 40224034.

Gerwick B. C. \& Sparks T. C. 2014. Natural products for pest control: an analysis of their role, value and future. Pest Management Science 70: 1169-1185.

Ikeda H., Nonomiya T., Usami M., Ohta T., \& Ōmura S. 1999. Organization of the biosynthetic gene cluster for the polyketide anthelmintic macrolide avermectin in Streptomyces avermitilis. Proceedings of the National Academy of Sciences USA. 96(17): 9509-95.

Jones A. C., Gust B., Kulik A., Heide L., Buttner M. J., \& Bibb M. J. 2013. Phage P1-derived artificial chromosomes facilitate heterologous expression of the FK506 gene cluster. PLoS ONE 8(7): e69319.

Kearse, M., Moir, R., Wilson, A., Stones-Havas, S., Cheung, M., Sturrock, S., Buxton, S., Cooper, A., Markowitz, S., Duran, C., Thierer, T., Ashton, B., Mentjies, P., \& Drummond, A. 2012. Geneious Basic: an integrated and extendable desktop software platform for the organization and analysis of sequence data. Bioinformatics 28(12): 1647-1649.

Kirst H. 2010. The spinosyn family of insecticides: realizing the potential of natural products research. Journal of Antibiotics 63: 101-111.

Kretschmer, A., Dorgerloh, M., Deeg, M., \& Hagenmaier, H. 1985. The structures of novel insecticidal macrolides: bafilomycins D and E, and oxohygrolidin. Agricultural and Biological Chemistry 49(8): 2509-2511.

Kwon M., Ahn Y. J., Yoo J. K., \& Choi B. R. 1996. Potent insecticidal activity of extracts from Ginkgo biloba leaves against Nilaparvata lugens (Homoptera: Delphacidae). Applied Entomology and Zoology 31: 162-166.

Langmead B. \& Salzberg S. 2012. Fast gapped-read alignment with Bowtie 2. Nature Methods 9: 357-359.

Luo Y., Cobb R. E., \& Zhao H. 2014. Recent advances in natural product discovery. Current Opinion in Biotechnology 30: 230237.

Seemann T. 2014. Prokka: rapid prokaryotic genome annotation. Bioinformatics 30(14): 2068-2069.

Shen, B. 2003. Polyketide biosynthesis beyond the type I, II and III polyketide synthase paradigms. Current Opinion in Chemical Biology 7: 285-295.

Shizuya H., Birren B., Kim U. J., Mancino V., Slepak T., Tachiiri Y., \& Simon M. 1992. Cloning and stable maintenance of 300-kilobase-pair fragments of human DNA in Escherichia coli using an F-factor-based vector. Proceedings of the National Academy of Sciences USA 89(18): 8794-8797.

Sosio M., Giusino F., Cappellano C., Bossi E., Puglia A. M., \& Donadio S. 2000. Artificial chromosomes for antibioticproducing actinomycetes. Nature Biotechnology 18(3): 343 345,2000

Sun Y., Zhou X., Bao K., Zhang G. et al. 2002. Streptomyces nanchangensis, a producer of the insecticidal polyetherantibiotic nanchangmycin and the antiparasitic macrolide meilingmycin, contains multiple polyketide gene clusters. Microbiology 36: 1-71.

Waldron, C., Madduri, K., Crawford, K. et al. 2000. A cluster of genes for the biosynthesis of spinosyns, novel macrolide insect control agents produced by Saccharopolyspora spinosa. Antonie Van Leeuwenhoek. 78: 385-390. 